Annals of Pure and Applied Mathematics

Vol. 18, No. 2, 2018, 139-146

ISSN: 2279-087X (P), 2279-0888(online)

Published on 31 October 2018

www.researchmathsci.org

DOI: http://dx.doi.org/10.22457/apam.v18n2a3

Annals of

Pure and Applied

Mathematics

\title{
Dakshayani Indices
}

\author{
V.R.Kulli
}

Department of Mathematics

Gulbarga University, Gulbarga 585106, India

e-mail: vrkulli@gmail.com

Received 15 October 2018; accepted 30 October 2018

Abstract. We introduce new topological indices, the first and second Dakshayani indices, general Dakshayani index. The second Dakshayani index is a linear combination of the modified first Zagreb index and inverse degree. We initiate a study of these new invariants.

Keywords: Modified first Zagreb index, inverse degree, Dakshayani index, polycyclic aromatic hydrocarbon, benzenoid system.

AMS Mathematics Subject Classification (2010): 05C05, 05C07, 05C12, 05 C35

\section{Introduction}

Let $G=(V(G), E(G))$ be a finite, connected graph. The degree $d_{G}(u)$ of a vertex $u$ is the number of vertices adjacent to $u$. The complement $\bar{G}$ of $G$ is the graph with vertex set $V(G)$ in which two vertices are adjacent if they are not adjacent in $G$. Clearly $d_{\bar{G}}(u)=n-1-d_{G}(u)$, where $n$ is the number of vertices in $G$. For other undefined notations and terminology, the readers are referred to [1]. A topological index is a numerical parameter mathematically derived from the graph structure.

In [2], Miličević et al. proposed the modified first Zagreb index, which is defined as

$$
{ }^{m} M_{1}(G)=\sum_{u \in V(G)} \frac{1}{d_{G}(u)^{2}} .
$$

This index was studied in [3].

The inverse degree is defined as

$$
I D(G)=\sum_{u \in V(G)} \frac{1}{d_{G}(u)} .
$$

This index has attracted through a conjecture of Grafitti[4]. The name inverse degree was proposed in [5].

We now introduce the first and second Dakshayani indices, defined as

$$
D K_{1}(G)=\sum_{u \in V(G)} d_{\bar{G}}(u) \frac{1}{d_{G}(u)},
$$




$$
D K_{2}(G)=\sum_{u \in V(G)} d_{\bar{G}}(u) \frac{1}{d_{G}(u)^{2}} .
$$
graph $G$ as

We continue this generalization and defined the general Dakshayani index of a

$$
D K^{a}(G)=\sum_{u \in V(G)} d_{\bar{G}}(u) d_{G}(u)^{a}
$$

where $a$ is a real number.

Clearly, $D K^{-1}(G)=D K_{1}(G), D K^{-2}(G)=D K_{2}(G)$.

Recently, many new topological indices were studied, for example, in $[6,7,8,9,10]$.

In section 2, we obtain exact value of the first and second Dakshayani indices for some standard graphs. In section 3, we determine some basic properties of $D K_{1}$ and $D K_{2}$. In section 4, the first and second Dakshayani indices and general Dakshayani index of polycyclic aromatic hydrocarbons and jagged rectangle benzenoid systems are computed.

\section{Results for some standard graphs}

The following are the first and second Dakshayani indices for paths, cycles, complete graphs, bipartite graphs.

Proposition 1. Let $P_{n}$ be a path with $n \geq 3$ vertices. Then

$$
\text { (i) } D K_{1}\left(P_{n}\right)=\frac{1}{2}(n-2)(n+1) \text {. (ii) } D K_{2}\left(P_{n}\right)=\frac{1}{4}(n-2)(n+5) \text {. }
$$

Proof: Let $G=P_{n}$. The vertex partition of $G$ is given in Table 1 .

\begin{tabular}{lll}
\hline$d_{G}(u) \backslash u \in V(G)$ & 1 & 2 \\
\hline$d_{\bar{G}}(u) \backslash u \in V(\bar{G})$ & $n-2$ & $n-3$ \\
\hline Number of vertices & 2 & $n-2$ \\
\hline
\end{tabular}

Table 1: Vertex partition of $P_{n}$

(i) $D K_{1}\left(P_{n}\right)=\sum_{u \in V(G)} d_{\bar{G}}(u) \frac{1}{d_{G}(u)}=\frac{(n-2) 2}{1}+\frac{(n-3)(n-2)}{2}=\frac{1}{2}(n-2)(n+1)$.

(ii) $D K_{2}\left(P_{n}\right)=\sum_{u \in V(G)} d_{\bar{G}}(u) \frac{1}{d_{G}(u)^{2}}=\frac{(n-2) 2}{1^{2}}+\frac{(n-3)(n-2)}{2^{2}}=\frac{1}{4}(n-2)(n+5)$.

Proposition 2. Let $C_{n}$ be a cycle with $n \geq 3$ vertices. Then
(i) $D K_{1}\left(C_{n}\right)=\frac{1}{2} n(n-3)$.
(ii) $D K_{2}\left(C_{n}\right)=\frac{1}{4} n(n-3)$.

Proof: Let $G=C_{n}$. The degree of each vertex of $C_{n}$ is 2 . Thus the degree each vertex of $\overline{C_{n}}$ is $(n-3)$. Thus

$$
D K_{1}\left(C_{n}\right)=\sum_{u \in V(G)} d_{\bar{G}}(u) \frac{1}{d_{G}(u)}=\frac{(n-3) n}{2} .
$$


Dakshayani Indices

(ii) $\quad D K_{2}\left(C_{n}\right)=\sum_{u \in V(G)} d_{\bar{G}}(u) \frac{1}{d_{G}(u)^{2}}=\frac{(n-3) n}{2^{2}}=\frac{1}{4} n(n-3)$.

Proposition 3. Let $K_{n}$ be a complete graph with $n \geq 3$ vertices. Then

(i) $D K_{1}\left(K_{n}\right)=0$.

Also $\quad D K_{1}\left(\overline{K_{n}}\right)=0$.

(ii) $D K_{2}\left(K_{n}\right)=0$.

Also $\quad D K_{2}\left(\overline{K_{n}}\right)=0$.

Proposition 4. Let $K_{m, n}$ be a complete bipartite graph with $1 \leq m<n$. Then

(1) $D K_{1}\left(K_{m, n}\right)=\frac{n(n-1)}{m}+\frac{m(m-1)}{n}$.

(2) $D K_{2}\left(K_{m, n}\right)=\frac{n(n-1)}{m^{2}}+\frac{m(m-1)}{n}$.

Proof: Let $G=K_{m, n}$. The vertex partition of $G$ is given in Table 2 .

\begin{tabular}{lll}
\hline$d_{G}(u) \backslash u \in V(G)$ & $m$ & $n$ \\
\hline$d_{\bar{G}}(u) \backslash u \in V(\bar{G})$ & $n-1$ & $m-1$ \\
\hline Number of vertices & $n$ & $m$ \\
\hline \multicolumn{2}{r}{ Table 2: Vertex partition of $K_{m, n}$}
\end{tabular}

Table 2: Vertex partition of $K_{m, n}$

(i) $\quad D K_{1}\left(K_{m, n}\right)=\sum_{u \in V(G)} d_{\bar{G}}(u) \frac{1}{d_{G}(u)}=\frac{(n-1) n}{m}+\frac{(m-1) m}{n}$.

(ii) $\quad D K_{2}\left(K_{m, n}\right)=\sum_{u \in V(G)} d_{\bar{G}}(u) \frac{1}{d_{G}(u)^{2}}=\frac{(n-1) n}{m^{2}}+\frac{(m-1) m}{n^{2}}$.

Corollary 4.1. Let $K_{1, n-1}$ be a star with $n \geq 2$ vertices. Then

(i) $D K_{1}\left(K_{1, n-1}\right)=(n-1)(n-2)$. (ii) $D K_{2}\left(K_{1, n-1}\right)=(n-1)(n-2)$.

Proposition 5. Let $G$ be an $r$-regular connected graph with $n \geq 2$ vertices. Then

(i) $D K_{1}(G)=\frac{1}{r} n(n-1-r) . \quad$ (ii) $D K_{2}(G)=\frac{1}{r^{2}} n(n-1-r)$.

Proof: The degree of every vertex of an $r$-regular graph $G$ is $r$. The degree every vertex of $\bar{G}$ is $n-1-r$.

$$
\begin{aligned}
& \text { (i) } D K_{1}(G)=\sum_{u \in V(G)} d_{\bar{G}}(u) \frac{1}{d_{G}(u)}=\frac{(n-1-r) n}{r} . \\
& \text { (ii) } D K_{2}(G)=\sum_{u \in V(G)} d_{\bar{G}}(u) \frac{1}{d_{G}(u)^{2}}=\frac{(n-1-r) n}{r^{2}} .
\end{aligned}
$$




\section{V.R.Kulli}

A double star $S_{p, q}$ is the graph obtained from joining the centers of two stars $K_{1, p-1}$ and $K_{1, q-1}$ with an edge.

Proposition 6. Let $S_{p, q}$ be a double star with $p+q$ vertices. Then

(i) $D K_{1}\left(S_{p, q}\right)=\frac{q-1}{p}+\frac{p-1}{q}+(p+q-2)^{2}$.

(ii) $D K_{2}\left(S_{p, q}\right)=\frac{q-1}{p^{2}}+\frac{p-1}{q^{2}}+(p+q-2)^{2}$.

Proof: Let $G=S_{p, q}$. The vertex partition of $G$ is given in Table 3 .

\begin{tabular}{llll}
\hline$d_{G}(u) \backslash u \in V(G)$ & $p$ & $q$ & 1 \\
\hline$d_{\bar{G}}(u) \backslash u \in V(\bar{G})$ & $q-1$ & $p-1$ & $p+q-2$ \\
\hline Number of vertices & 1 & 1 & $p+q-2$ \\
\hline
\end{tabular}

Table 3: Vertex partition of $S_{p, q}$

(i) $D K_{1}\left(S_{p, q}\right)=\sum_{u \in V(G)} d_{\bar{G}}(u) \frac{1}{d_{G}(u)}=\frac{q-1}{p}+\frac{p-1}{q}+(p+q-2)^{2}$.

(ii) $D K_{2}\left(S_{p, q}\right)=\sum_{u \in V(G)} d_{\bar{G}}(u) \frac{1}{d_{G}(u)^{2}}=\frac{q-1}{p^{2}}+\frac{p-1}{q^{2}}+(p+q-2)^{2}$.

Corollary 6.1. Let $S_{\frac{n}{2}, \frac{n}{2}}$ be a double star with $n$ vertices. Then,

(i) $D K_{1}\left(S_{\frac{n}{2}, \frac{n}{2}}\right)=n^{2}-4 n-\frac{2}{n}+6$.

(ii) $D K_{2}\left(S_{\frac{n}{2}, \frac{n}{2}}\right)=n^{2}-4 n+\frac{4}{n}-\frac{8}{n^{2}}+4$.

Proof: (i) Put $p=\frac{n}{2}, q=\frac{n}{2}$ in equation (4), we get the desired result.

(ii) Put $p=\frac{n}{2}, q=\frac{n}{2}$ in equation (5), we get the desired result.

Proposition 7. Let $G$ be a graph with $n$ vertices. Then (i) $0 \leq D K_{1}(G)$ and (ii) $0 \leq$ $D K_{2}(G)$. In (i) and (ii), equality holds if and only if $G=K_{n}$ or $\overline{K_{n}}$.

\section{Results on Dakshayani indices}

In this section, we investigate some mathematical properties of Dakshayani indices.

Proposition 8. Let $G$ be a simple connected graph. Then $D K_{1}(G)=(n-1) I D(G)-n$.

Proof: Follows from equation (1). 
Dakshayani Indices

Proposition 9. Let $G$ be a simple connected graph. Then $n \leq(n-1) I D(G)$.

Proof: This follows from propositions 7(i) and 8.

Proposition 10. Let $G$ be a simple connected graph. Then

$$
D K_{2}(G)=(n-1)^{m} M_{1}(G)-I D(G) \text {. }
$$

Proof: We have

$$
\begin{aligned}
D K_{2}(G) & =\sum_{u \in V(G)} d_{\bar{G}}(u) \frac{1}{d_{G}(u)^{2}}=\sum_{u \in V(G)}\left[n-1-d_{G}(u)\right] \frac{1}{d_{G}(u)^{2}} \\
& =\sum_{u \in V(G)}\left[(n-1) \frac{1}{d_{G}(u)^{2}}-\frac{1}{d_{G}(u)}\right]
\end{aligned}
$$

Thus $D K_{2}(G)=(n-1)^{m} M_{1}(G)-I D(G)$.

Therefore the second Dakshayani index is a linear combination of the modified first Zagreb index and inverse degree.

Proposition 11. Let $G$ be a simple connected graph. Then

(i) ${ }^{m} M_{1}(G)=\frac{1}{n-1}\left[D K_{2}(G)+I D(G)\right]$.

(ii) $I D(G)=(n-1)^{m} M_{1}(G)-D K_{2}(G)$.

Proof: These follow from propositions 7(ii) and 10.

\section{Results for some chemical compounds}

\subsection{Results for polycyclic aromatic hydrocarbons}

In this section, we focus on the chemical graph structure of the family of polycyclic aromatic hydrocarbons, denoted by $P A H_{n}$. The first three members of the family $P A H_{n}$ are given in Figure 1.

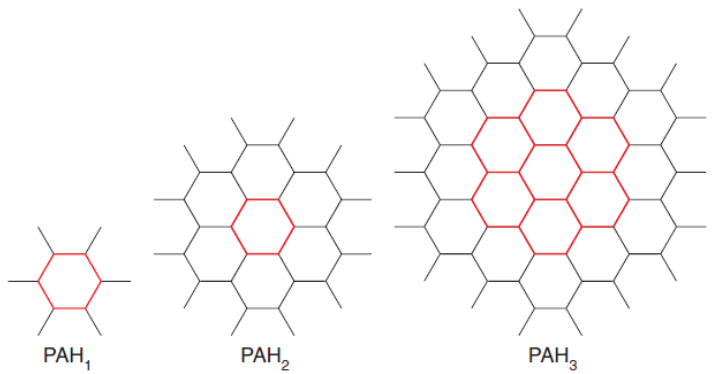

Figure 1:

Let $G=P A H_{n}$ be the chemical graph in family of polycyclic aromatic hydrocarbons. By calculation, we obtain that $G$ has $6 n^{2}+6 n$ vertices. There are two types of vertices in $G$ and hence $\bar{G}$ has two types of vertices as given in Table 4 . 
V.R.Kulli

\begin{tabular}{lll}
\hline$d_{G}(u) \backslash u \in V(G)$ & 1 & 3 \\
\hline$d_{\bar{G}}(u) \backslash u \in V(\bar{G})$ & $6 n^{2}+6 n-2$ & $6 n^{2}+6 n-4$ \\
\hline Number of vertices & $6 n$ & $6 n^{2}$ \\
\hline
\end{tabular}

Table 4: Vertex partitions of $G$ and $\bar{G}$.

Theorem 1. The first and second Dakshayani indices of the family of polycyclic aromatic hydrocarbons $P A H_{n}$ are

(i) $D K_{1}\left(P A H_{n}\right)=12 n^{4}+48 n^{3}+28 n^{2}-12 n$.

(ii) $D K_{2}\left(P A H_{n}\right)=4 n^{4}+40 n^{3}+\frac{100}{3} n^{2}-12 n$.

Proof: Let $G=P A H_{n}$ be the chemical graph in the family of polycyclic aromatic hydrocarbons.

(i) From equation (1) and using Table 1, we deduce

$$
\begin{aligned}
D K_{1}\left(P A H_{n}\right) & =\sum_{u \in V(G)} d_{\bar{G}}(u) \frac{1}{d_{G}(u)} \\
& =\left[\left(6 n^{2}+6 n-2\right) \frac{1}{1}\right] 6 n+\left[\left(6 n^{2}+6 n-4\right) \frac{1}{3}\right] 6 n^{2} \\
& =12 n^{4}+48 n^{3}+28 n^{2}-12 n .
\end{aligned}
$$

(ii) From equation (2) and using Table 1, we deduce

$$
\begin{aligned}
D K_{2}\left(P A H_{n}\right) & =\sum_{u \in V(G)} d_{\bar{G}}(u) \frac{1}{d_{G}(u)^{2}} \\
& =\left[\left(6 n^{2}+6 n-2\right) \frac{1}{1^{2}}\right] 6 n+\left[\left(6 n^{2}+6 n-4\right) \frac{1}{3^{2}}\right] 6 n^{2} \\
& =4 n^{4}+40 n^{3}+\frac{100}{3} n^{2}-12 n .
\end{aligned}
$$

Theorem 2. The general Dakshayani index of the family of polycyclic aromatic hydrocarbons $P A H_{n}$ is

$$
D K^{a}\left(P A H_{n}\right)=6 n\left(6 n^{2}+6 n-2\right)+6 \times 3^{a} n^{2}\left(6 n^{2}+6 n-4\right)
$$

Proof: Let $G=P A H_{n}$. By using equation (3) and Table 1, we obtain

$$
\begin{aligned}
D K^{a}\left(P A H_{n}\right) & =\sum_{u \in V(G)} d_{\bar{G}}(u) d_{G}(u)^{a} \\
& =\left[\left(6 n^{2}+6 n-2\right) 1^{a}\right] 6 n+\left[\left(6 n^{2}+6 n-4\right) 3^{a}\right] 6 n^{2} \\
& =\left(6 n^{2}+6 n-2\right) 6 n+3^{a}\left(6 n^{2}+6 n-4\right) 6 n^{2} .
\end{aligned}
$$

\subsection{Results for Benzenoid systems}

In this section, we focus on the chemical graph structure of a jagged rectangle benzenoid system, denoted by $B_{m, n}$ for all $m, n \in N$. Three chemical graphs of jagged rectangle benzenoid systems are presented in Figure 2. 
Dakshayani Indices
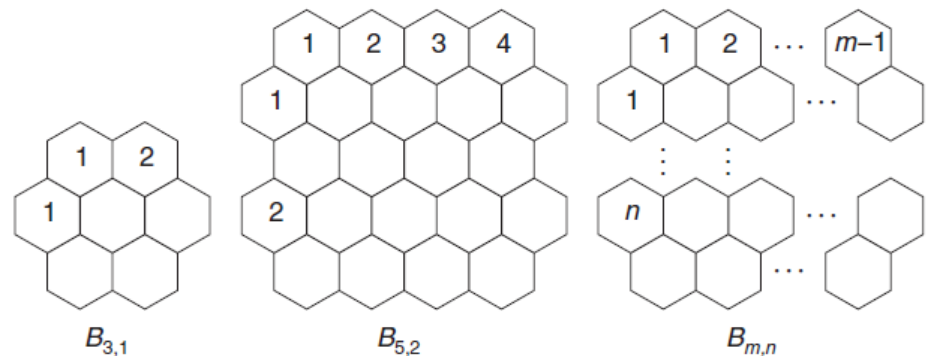

Figure 2:

Let $G=B_{m, n}$ be the graph in the family of a jagged rectangle benzenoid system. By calculation, we obtain that $G$ has $4 m n+4 m+2 n-2$ vertices. The graph $G$ has two types of vertices and hence $\bar{G}$ has two types of vertices as given in Table 5 .

\begin{tabular}{lll}
\hline$d_{G}(u) \backslash u \in V(G)$ & 2 & 3 \\
\hline$d_{\bar{G}}(u) \backslash u \in V(\bar{G})$ & $4 m n+4 m+2 n-3$ & $4 m n+4 m+2 n-4$ \\
\hline Number of vertices & $2 m+4 n+2$ & $4 m n+2 m-2 n-4$ \\
\hline
\end{tabular}

Table 5: Vertex partitions of $G$ and $\bar{G}$.

Theorem 3. The first and second Dakshayani indices of the family of jagged benzenoid systems $B_{m, n}$ are

(i) $D K_{1}\left(B_{m, n}\right)=\frac{16}{3} m^{2} n^{2}+12 m^{2} n+8 m n^{2}+2 m n+\frac{20}{3} m^{2}-7 m+\frac{8}{3} n^{2}-4 n+\frac{7}{3}$

(ii) $D K_{2}\left(B_{m, n}\right)=\frac{16}{9} m^{2} n^{2}+\frac{42}{9} m^{2} n+4 m n^{2}+3 m n+\frac{26}{9} m^{2}-\frac{13}{6} m+\frac{14}{9} n^{2}-2 n+\frac{5}{18}$.

Proof: Let $G=B_{m, n}$.

(i) From equation (1) and using Table 2, we derive

$$
\begin{aligned}
D K_{1}\left(B_{m, n}\right) & =\sum_{u \in V(G)} d_{\bar{G}}(u) \frac{1}{d_{G}(u)} \\
& =\left[(4 m n+4 m+2 n-3) \frac{1}{2}\right](2 m+4 n+2) \\
& +\left[(4 m n+4 m+2 n-4) \frac{1}{3}\right](4 m n+2 m-2 n-4) \\
& =\frac{16}{3} m^{2} n^{2}+12 m^{2} n+8 m n^{2}+2 m n+\frac{20}{3} m^{2}-7 m+\frac{8}{3} n^{2}-4 n+\frac{7}{3}
\end{aligned}
$$

(ii) From equation (2) and using Table 2, we derive

$$
\begin{aligned}
D K_{2}\left(B_{m, n}\right) & =\sum_{u \in V(G)} d_{\bar{G}}(u) \frac{1}{d_{G}(u)^{2}} \\
& =\left[(4 m n+4 m+2 n-3) \frac{1}{2^{2}}\right](2 m+4 n+2)
\end{aligned}
$$




$$
\begin{aligned}
& +\left[(4 m n+4 m+2 n-4) \frac{1}{3^{2}}\right](4 m n+2 m-2 n-4) \\
& =\frac{16}{9} m^{2} n^{2}+\frac{42}{9} m^{2} n+4 m n^{2}+3 m n+\frac{26}{9} m^{2}-\frac{13}{6} m+\frac{14}{9} n^{2}-2 n+\frac{5}{18}
\end{aligned}
$$

Theorem 4. The general Dakshayani index of the family of jagged rectangle benzenoid systems $B_{m, n}$ is

$$
\begin{aligned}
D K^{a}\left(B_{m, n}\right) & =2^{a}(4 m n+4 m+2 n-3)(2 m+4 n-2) \\
& +3^{a}(4 m n+4 m+2 n-4)(4 m n+2 m-2 n-4) .
\end{aligned}
$$

Proof: Let $G=B_{m, n}$. By using equation (3) and Table 2, we deduce

$$
\begin{aligned}
D K^{a}\left(B_{m, n}\right) & =2^{a}(4 m n+4 m+2 n-3)(2 m+4 n-2) \\
& +3^{a}(4 m n+4 m+2 n-4)(4 m n+2 m-2 n-4) .
\end{aligned}
$$

Acknowledgement. The author is thankful to the referee for useful suggestions.

\section{REFERENCES}

1. V.R.Kulli, College Graph Theory, Vishwa International Publications, Gulbarga, India (2012).

2. A.Milićević and S. Nikolić On variable Zagreb indices, Croat. Chem Acta, 77 (2004) 97-101.

3. V.R.Kulli, Some topological indices of certain nanotubes, Journal of Computer and Mathematical Sciences, 8(1) (2017) 1-7.

4. S. Fajtolowicz, On conjectures of Grafitti, Discrete Math., 72 (1988) 113-118.

5. Z.Zhang, J.Zhang and X.Lu, The relation of matching with inverse degree of a graph, Discrete Math. 301(2005) 243-246.

6. V.R.Kulli, The Gourava indices and coindices of graphs, Annals of Pure and Applied Mathematics, 14(1) (2017) 33-38.

7. V.R.Kulli, Computation of F-reverse index and modified reverse indices of some nanostructures, Annals of Pure and Applied Mathematics, 18(1) (2018) 37-43.

8. V.R.Kulli, On reduced Zagreb indices of polycyclic aromatic hydrocarbons and benzenoid systems, Annals of Pure and Applied Mathematics, 18(1) (2018) 73-78.

9. S.Ediz, Maximal graphs of the first reverse Zagreb beta index, TWMS J. App. Eng. Math. accepted for publication.

10. V.R.Kulli, B.Stone, S.Wang and B.Wei, Generalized multiplicative indices of polycyclic aromatic hydrocarbons and benzenoid systems, Z. Naturforsch, 72(6) a (2017) 573-576. 